\title{
Dark Higgs dark matter
}

\author{
Cristina Mondino®, ${ }^{1,2}$ Maxim Pospelov, ${ }^{3}$ Joshua T. Ruderman, ${ }^{1}$ and Oren Slone ${ }^{4}$ \\ ${ }^{1}$ Center for Cosmology and Particle Physics, Department of Physics, New York University, \\ New York, New York 10003, USA \\ ${ }^{2}$ Perimeter Institute for Theoretical Physics, Waterloo, Ontario N2L 2Y5, Canada \\ ${ }^{3}$ William I. Fine Theoretical Physics Institute, School of Physics and Astronomy, \\ University of Minnesota, Minneapolis, Minnesota 55455, USA \\ ${ }^{4}$ Princeton Center for Theoretical Science, Princeton University, Princeton, New Jersey 08544, USA
}

(Received 4 June 2020; revised 16 January 2021; accepted 27 January 2021; published 25 February 2021)

A new $U(1)$ "dark" gauge group coupled to the Standard Model (SM) via the kinetic mixing portal provides a dark matter candidate in the form of the Higgs field, $h_{d}$, responsible for generating the mass of the dark photon, $\gamma_{d}$. We show that the condition $m_{h_{d}} \leq m_{\gamma_{d}}$, together with smallness of the kinetic mixing parameter, $\epsilon$, and/or dark gauge coupling, $g_{d}$, leads the dark Higgs to be sufficiently metastable to constitute dark matter. We analyze the Universe's thermal history and show that both freeze-in, SM $\rightarrow\left\{\gamma_{d}, h_{d}\right\}$, and freeze-out, $\left\{\gamma_{d}, h_{d}\right\} \rightarrow \mathrm{SM}$, processes can lead to viable dark Higgs dark matter with a sub-GeV mass and a kinetic mixing parameter in the range $10^{-13} \lesssim \epsilon \lesssim 10^{-6}$. Observable signals in astrophysics and cosmology include modifications to primordial elemental abundances, altered energetics of supernovae explosions, dark Higgs decays in the late Universe, and dark matter self-interactions.

DOI: 10.1103/PhysRevD.103.035027

\section{INTRODUCTION}

Evidence for Dark Matter (DM) constitutes a strong argument for extending the Standard Model (SM) with Dark Sector(s) (DS). A simple example is an additional $U(1)_{d}$ gauge symmetry associated with its gauge boson, the dark photon $\gamma_{d}$, that mediates DS-SM interactions. It is commonly assumed that DM is charged under $U(1)_{d}$ and is represented by additional states in the DS.

Much theoretical and experimental attention has been devoted to the study of such a DS [1,2] in recognition of the fact that its mass scale can be at, or below $1 \mathrm{GeV}$, offering a variety of new probes. In this study, we show that an even more minimal option exists: the field responsible for generating the mass of $\gamma_{d}$, a dark Higgs $h_{d}$, is a viable DM candidate with different phenomenological implications than those commonly assumed [1,2]. We analyze the salient features of Dark Higgs Dark Matter (DHDM), including its (meta)stability and genesis through cosmic history.

The relevant DS Lagrangian is

$$
\mathcal{L}_{\mathrm{DS}}=\left|D^{\mu} \phi\right|^{2}-\frac{1}{4}\left(F_{d}^{\mu \nu}\right)^{2}-\frac{\epsilon}{2} F_{d}^{\mu \nu} F_{\mu \nu}-V(\phi),
$$

Published by the American Physical Society under the terms of the Creative Commons Attribution 4.0 International license. Further distribution of this work must maintain attribution to the author(s) and the published article's title, journal citation, and DOI. Funded by SCOAP ${ }^{3}$. where $\phi$ is a charged scalar field, $F^{\mu \nu}$ and $F_{d}^{\mu \nu}$ are the SM and dark photon field strengths, and $\epsilon$ is the kinetic mixing parameter. The scalar potential, $V(\phi)=-\mu^{2}|\phi|^{2}+\lambda|\phi|^{4}$, generates a nonzero Vacuum Expectation Value (VEV), $\langle\phi\rangle=\mu / \sqrt{2 \lambda} \equiv v / \sqrt{2}$, that spontaneously breaks the $U(1)_{d}$ gauge symmetry. Expanding $\phi$ around the VEV, $\phi=\left(v+h_{d}\right) / \sqrt{2}$, generates the mass terms $m_{h_{d}}=\sqrt{2 \lambda} v$ and $m_{\gamma_{d}}=g_{d} v$, where $g_{d}$ is the dark gauge coupling, and one can define $\alpha_{d} \equiv g_{d}^{2} / 4 \pi$.

For natural couplings, $\lambda \sim g_{d}^{2}$, the dark particle's masses are of similar order. If $m_{h_{d}} \lesssim m_{\gamma_{d}}, h_{d}$ has a potentially long lifetime $[3,4]$. Then, the only open decay channels for an $\mathrm{MeV}$-scale dark Higgs are $h_{d} \rightarrow e^{+} e^{-} e^{+} e^{-}$and $h_{d} \rightarrow e^{+} e^{-}$ (see Fig. 1), inducing a dark Higgs lifetime which scales as $\tau_{h_{d}} \propto \epsilon^{-4}$ (for full expressions see e.g., Ref. [3]). An additional Higgs portal coupling could lead to further decay channels. However, if absent at tree level, this coupling only appears at one loop, resulting in a negligibly small SM-DS Higgs mixing parameter, $\theta_{h-h_{d}} \propto\left(\epsilon g_{d}\right)^{2}\left(v / v_{\mathrm{EW}}\right)$.

Combining the decay channels for $4 m_{e} \ll m_{h_{d}}<2 m_{\mu}$, the approximate decay width for DHDM is

$$
\tau_{U} \Gamma_{2 e, 4 e} \simeq 8 \times 10^{-8}\left[\frac{\epsilon}{10^{-9}}\right]^{4}\left[\frac{\alpha_{d}}{10^{-4}}\right]\left[\frac{m_{h_{d}}}{100 \mathrm{MeV}}\right] f .
$$

Here, $\tau_{U}$ is the age of the Universe, and $f=f\left(m_{h_{d}} / m_{\gamma_{d}}\right)$ is of order unity at $m_{h_{d}}=m_{\gamma_{d}}$. Note that $\tau_{U} \Gamma_{2 e, 4 e}<1$ is an insufficient condition for $h_{d}$ to be DM, since stronger 


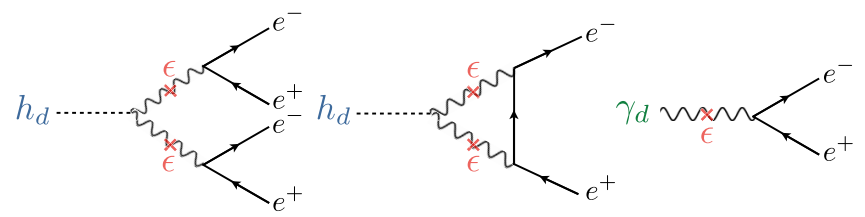

FIG. 1. Decay processes of dark sector particles. Left and central diagrams are dark Higgs decay channels available for $4 m_{e}<m_{h_{d}}<2 m_{\mu}$ and $m_{h_{d}}<m_{\gamma_{d}}$. The right diagram is the dark photon decay channel for $2 m_{e}<m_{\gamma_{d}}<2 m_{\mu}$.

bounds are imposed by limits on diffuse photon spectra [5] and measurements of $\mathrm{CMB}$ anisotropies [6,7], requiring $1 / \Gamma_{2 e, 4 e} \gtrsim 10^{25} s$.

\section{BRIEF OVERVIEW OF DHDM COSMIC HISTORY}

Various processes affect the DS's thermal history. Those that exchange energy between the two sectors have rates that scale as $\epsilon^{2}$. Of these, dark photon decay and inverse decay, $\gamma_{d} \leftrightarrow e^{+} e^{-}$(see Fig. 1), always dominate over the scattering processes $\gamma_{d} e^{ \pm} \leftrightarrow \gamma e^{ \pm}, \quad h_{d} \gamma_{d} \leftrightarrow e^{+} e^{-}$, and $h_{d} e^{ \pm} \leftrightarrow \gamma_{d} e^{ \pm}$. Interactions involving only DS particles are controlled by $\alpha_{d}$. Both $2 \leftrightarrow 2$ and $3 \leftrightarrow 2$ processes are relevant, with rates that scale as $\alpha_{d}^{2}$ and $\alpha_{d}^{3}$, respectively (see Fig. 2). Given these ingredients, the DS is described by the parameters $\left\{m_{\gamma_{d}}, m_{h_{d}}, \alpha_{d}, \epsilon\right\}$.

We identify three regimes of interest, schematically represented in Fig. 3 and corresponding to different production mechanisms and phenomenology. When $\epsilon \sim 10^{-13}-10^{-11}$, the DS is thermally decoupled from the SM and production of DHDM proceeds via freeze-in of $\gamma_{d}$, which annihilate into $h_{d}$. Alternatively, the DS can be independently populated during reheating, reaching internal thermal equilibrium (if $\alpha_{d}$ is sufficiently large). For $\epsilon \sim 10^{-11}-10^{-8}$, the DS never fully thermalizes with the SM. However, $h_{d}$ can deplete its number density by annihilating into $\gamma_{d}$ that decay into SM particles. Finally, going to $\epsilon \gtrsim 10^{-8}$ requires increasing $\tau_{h_{d}}$ by choosing a very small $\alpha_{d}$. In this case, the dark photon reaches thermal equilibrium with the $\mathrm{SM}$, and $h_{d}$ can freeze-in from the thermal bath of $\gamma_{d}$. An upper limit of $\epsilon \lesssim 10^{-6}$ follows from constraints on $\tau_{h_{d}}$.

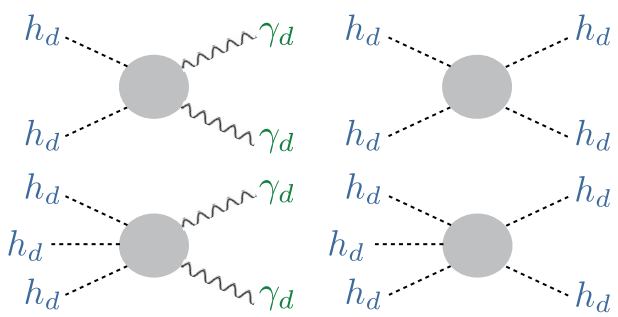

FIG. 2. Diagrams for processes involving dark sector particles only. Rates of $2 \leftrightarrow 2$ processes on the top row scale as $\alpha_{d}^{2}$ while $3 \leftrightarrow 2$ processes on the bottom row scale as $\alpha_{d}^{3}$.

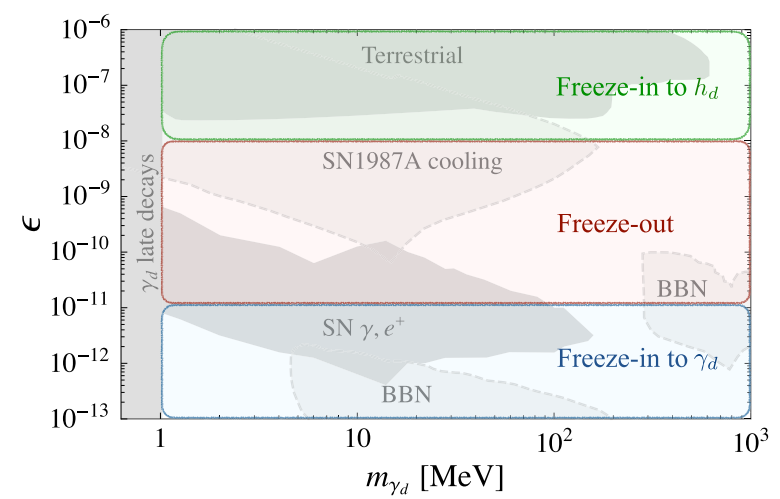

FIG. 3. Illustration of various DHDM production regimes: freeze-in to $\gamma_{d}$ followed by annihilation to $h_{d}$ at tiny kinetic mixing, freeze-out of secluded annihilation at intermediate values, and freeze-in to $h_{d}$ with $\gamma_{d}$ in equilibrium with the SM at large $\epsilon$. The true boundaries between the regimes are $m_{\gamma_{d}}$ and $m_{h_{d}}$ dependent. Gray shaded regions show existing bounds on the dark photon, reproduced from Refs. [8-10]. Lightly shaded regions are potentially modified by the presence of the dark Higgs.

Assuming a thermal distribution for the DS particles, the above regimes can be described with a set of three coupled Boltzmann Equations (BEs). The first two, 0th moment BEs, track the number densities,

$\dot{n}_{h_{d}}+3 H n_{h_{d}}=\mathcal{C}_{2 \gamma_{d} \rightarrow 2 h_{d}}-\mathcal{C}_{3 h_{d} \rightarrow 2 \gamma_{d}}$,
$\dot{n}_{\gamma_{d}}+3 H n_{\gamma_{d}}=-\mathcal{C}_{\gamma_{d} \rightarrow e^{+} e^{-}}-\mathcal{C}_{2 \gamma_{d} \rightarrow 2 h_{d}}+\frac{2}{3} \mathcal{C}_{3 h_{d} \rightarrow 2 \gamma_{d}}$,

where $H$ is the Hubble rate, and the collision terms, $\mathcal{C}_{\text {init } \rightarrow \text { fin }}$, correspond to the processes of Figs. 1 and 2 (explicit expressions appear in Appendix A). The third, 1st moment $\mathrm{BE}$, tracks the DS temperature, $T_{d}$. Defining $x_{d} \equiv m_{h_{d}} / T_{d}, r \equiv m_{\gamma_{d}} / m_{h_{d}}$, and $n \equiv n_{\gamma_{d}}+n_{h_{d}}$,

$$
\begin{aligned}
& {\left[n_{h_{d}} F^{\prime}\left(x_{d}\right)+r^{2} n_{\gamma_{d}} F^{\prime}\left(r x_{d}\right)\right] \dot{x}_{d}+\frac{3 H n}{x_{d}}} \\
& \quad=-\mathcal{C}_{\gamma_{d} \rightarrow e^{+} e^{-}}^{(E)}+\mathcal{C}_{2 \gamma_{d} \rightarrow 2 h_{d}}^{(E)}+\mathcal{C}_{3 h_{d} \rightarrow 2 \gamma_{d}}^{(E)},
\end{aligned}
$$

where $F(x)=K_{1}(x) / K_{2}(x)+3 / x$, and $K_{1,2}$ denote the modified Bessel functions. In the nonrelativistic limit, when $T_{d} \lesssim m_{h_{d}}, m_{\gamma_{d}}$, the above equation reduces to

$$
n \frac{\dot{T_{d}}}{T_{d}}+2 H n=\mathcal{C}_{\gamma_{d} \rightarrow e^{+} e^{-}}^{(E), \mathrm{nr}}+\mathcal{C}_{2 \gamma_{d} \rightarrow 2 h_{d}}^{(E), \mathrm{nr}}+\frac{2}{3} \mathcal{C}_{3 h_{d} \rightarrow 2 \gamma_{d}}^{(E), \mathrm{nr}}
$$

In Eqs. (3)-(6), only dominant processes have been included. A full derivation is presented in Appendix A.

\section{FREEZE-IN REGIMES}

At extremely small $\epsilon$, the DS is very weakly coupled to the SM. Assuming negligible production of DS particles 
during reheating (setting the initial $n_{h_{d}}=n_{\gamma_{d}}=0$ ), dark photons can freeze-in from SM particles via out-of-equilibrium processes [8,11-13], dominated by inverse decay. After a population of $\gamma_{d}$ develops, these annihilate exothermically into dark Higgs through $\gamma_{d} \gamma_{d} \rightarrow h_{d} h_{d}$. The result of such a freeze-in to $\gamma_{d}$ scenario is shown in Fig. 4, for $m_{h_{d}}=2 \mathrm{MeV}$. At every point, the ratio $r$ is chosen to match the observed DM relic density, $\Omega_{h_{d}} h^{2}=0.12$ [14] (when multiple $r$ values satisfy this condition, the lowest has been chosen). Three qualitatively different regimes occur, delineated by dashed green lines.

Two of these regimes occur when $\alpha_{d}$ is small enough such that $2 \leftrightarrow 3$ processes are negligible. Then, the total DS number density, $n$, can be calculated by summing Eqs. (3) and (4). This results in the following yield, $Y \equiv$ $n / s$ ( $s$ is the entropy density of the Universe), at late times,

$$
Y \approx \frac{3 m_{\gamma_{d}}^{3} \Gamma_{\gamma_{d}}^{0}}{2 \pi^{2} r} \int_{x \ll 1}^{x \approx 10} \frac{K_{1}(r x)}{x^{2} H s} d x \approx 7 \times 10^{-5} \frac{\epsilon^{2} m_{\mathrm{pl}}}{m_{\gamma_{d}}},
$$

where $x \equiv m_{h_{d}} / T_{\mathrm{SM}}, \quad T_{\mathrm{SM}}$ is the $\mathrm{SM}$ temperature, $\Gamma_{\gamma_{d}}^{0} \approx \alpha \epsilon^{2} m_{\gamma_{d}} / 3$ with $\alpha \simeq 1 / 137$, and we have taken

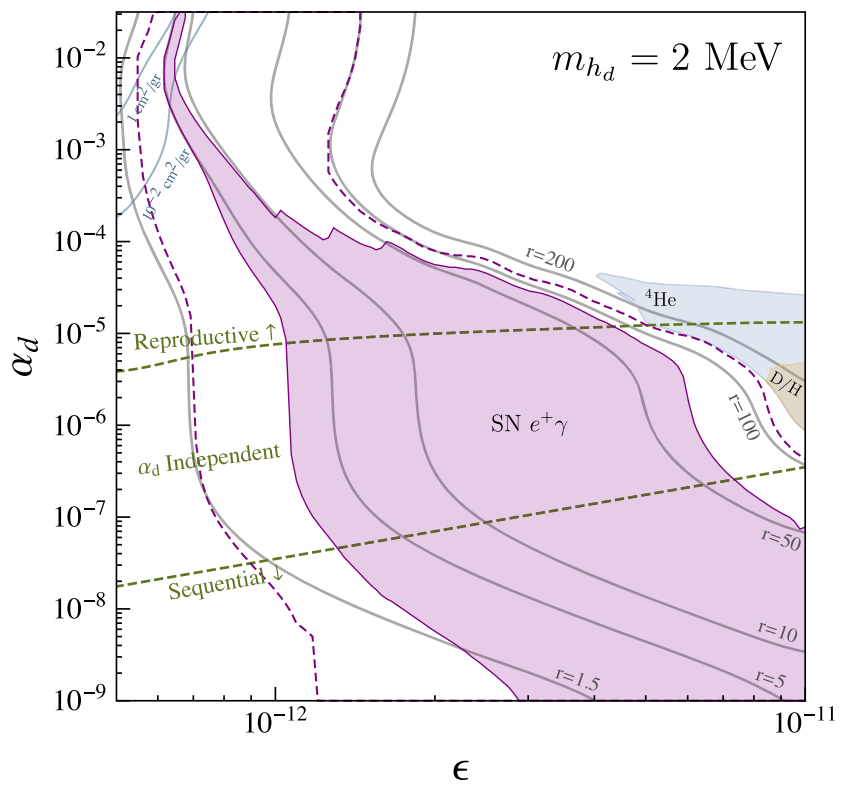

FIG. 4. Parameter space for the freeze-in to dark photon regime. The correct DHDM relic density is produced everywhere by fixing $r \equiv m_{\gamma_{d}} / m_{h_{d}}$; constant values are shown as gray contours. Three qualitatively different regions are evident: sequential freeze-in where only a fraction of $\gamma_{d}$ annihilate into $h_{d}$, an $\alpha_{d}$ independent regime where essentially all $\gamma_{d}$ annihilate into $h_{d}$, and a reproductive regime where $2 \rightarrow 3$ processes become important. Also shown are bounds from the nonobservation of $e^{+}$and $\gamma$ signals of $\gamma_{d}$ production within SNe [10] (discovery potential given by the purple dashed line), and Big Bang Nucleosynthesis (BBN) constraints from overproduction of ${ }^{4} \mathrm{He}$ and D [15]. Blue contours correspond to constant selfinteraction cross sections per unit mass, $\sigma_{\mathrm{SIDM}} / m_{h_{d}}$. $g_{*} \approx g_{* s} \approx 10$. The final yield of $h_{d}$ depends on the efficiency of the annihilation process, $\gamma_{d} \gamma_{d} \rightarrow h_{d} h_{d}$, which is controlled by $r$ and $\alpha_{d}$.

For a given $r$, when $\alpha_{d}$ is small enough such that most dark photons do not annihilate, the result can be approximated by taking $Y \rightarrow Y_{\gamma_{d}}$ in Eq. (7), and plugging into Eq. (3) while setting $\mathcal{C}_{3 h_{d} \rightarrow 2 \gamma_{d}}=0$. Then, the rate for generating DHDM from $\gamma_{d}$ scales as $\propto Y_{\gamma_{d}}^{2} \alpha_{d}^{2} m_{\gamma_{d}}^{-2}$. Neglecting inverse annihilation, one finds

$\frac{\Omega_{h_{d}} h^{2}}{0.12} \approx\left[\frac{\epsilon}{5 \times 10^{-12}}\right]^{4}\left[\frac{\alpha_{d}}{10^{-9}}\right]^{2}\left[\frac{m_{h_{d}}}{2 \mathrm{MeV}}\right]\left[\frac{4 \mathrm{MeV}}{m_{\gamma_{d}}}\right]^{3}$.

Thus, the $h_{d}$ relic density is fixed along contours of constant $\epsilon^{4} \alpha_{d}^{2}$. This is a realization of the sequential freeze-in mechanism, identified for a different model by Ref. [16].

For larger $\alpha_{d}, \gamma_{d} \gamma_{d} \rightarrow h_{d} h_{d}$ annihilations become efficient and essentially all dark photons convert into DHDM. The abundance becomes $\alpha_{d}$ independent and can be approximated by replacing $Y \rightarrow Y_{h_{d}}$ in Eq. (7),

$$
\frac{\Omega_{h_{d}} h^{2}}{0.12} \approx\left[\frac{\epsilon}{10^{-12}}\right]^{2}\left[\frac{m_{h_{d}}}{2 \mathrm{MeV}}\right]\left[\frac{4 \mathrm{MeV}}{m_{\gamma_{d}}}\right] .
$$

The third regime occurs when $\alpha_{d}$ becomes large enough that number changing processes within the DS are important. A particularly interesting phenomenon occurs when the rate for $\gamma_{d} \gamma_{d} \leftrightarrow h_{d} h_{d}$ is faster than Hubble, and $2 \rightarrow 3$ processes are simultaneously active. The former imposes chemical equilibrium between $h_{d}$ and $\gamma_{d}$, whereas the latter pushes their initially negative chemical potentials to zero. This combination causes a rapid drop in $T_{d}$ via removal of kinetic energy, setting $n_{\gamma_{d}} \approx n_{\gamma_{d}}^{\mathrm{eq}}$ and $n_{h_{d}} \approx n_{h_{d}}^{\mathrm{eq}}$, while washing out any dependence on the initial $T_{d}$. A similar regime has previously been studied for different models in Refs. [17-21]. We denote this production mechanism reproductive freeze-in, and provide further details in Appendix B. An example of numerical solutions for $n_{\gamma_{d}}$, $n_{h_{d}}$, and $T_{d}$, in the reproductive regime, is shown in Fig. 5. Production of dark particles from the $2 \rightarrow 3$ processes causes the gray contours in Fig. 4 to move towards smaller $\epsilon$.

As discussed above, freeze-in can also occur for $\epsilon \gtrsim 10^{-8}$, when dark photons are in thermal equilibrium with the SM. For $\alpha_{d} \sim 10^{-13}$, dark Higgs particles freezein from the $\left\{\mathrm{SM}, \gamma_{d}\right\}$ plasma, through the processes $\gamma_{d} \gamma_{d} \rightarrow h_{d} h_{d}, e^{+} e^{-} \rightarrow \gamma_{d} h_{d}$, and $e \gamma_{d} \rightarrow e h_{d}$. A similar scenario for freeze-in of the Higgs field of a $U(1)_{B-L}$ symmetry has recently been considered in Ref. [22].

\section{A. Phenomenological consequences}

The phenomenology of the freeze-in to $\gamma_{d}$ scenario is summarized in Fig. 4. Dark photons produced within supernovae $(\mathrm{SNe})$ can decay outside the explosion and 


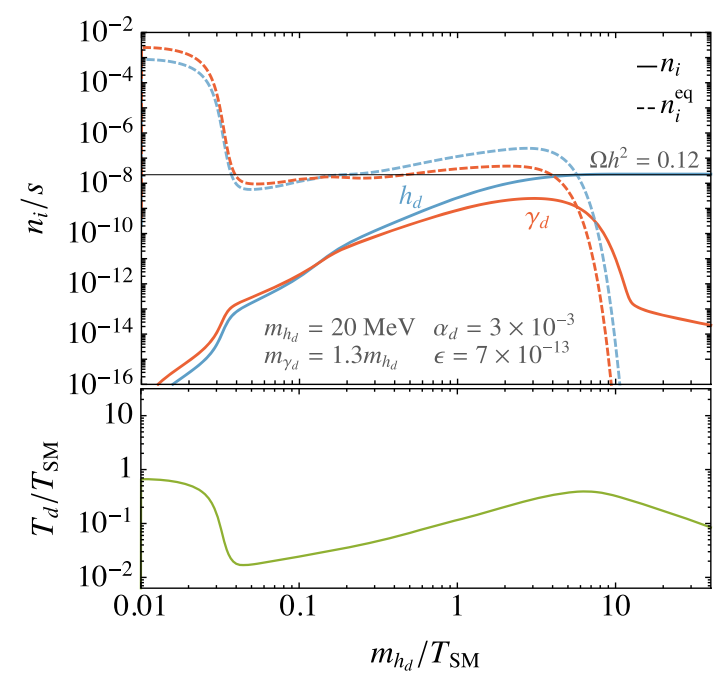

FIG. 5. Example of the DS evolution in the reproductive freezein regime. The top panel shows the evolution of $h_{d}$ and $\gamma_{d}$ comoving number densities. The bottom panel shows the temperature ratio, $T_{d} / T_{\mathrm{SM}}$. Dashed curves correspond to equilibrium distributions.

produce positron annihilation or gamma-ray signals [10]. Nonobservation of these signals excludes a region in the $\left\{m_{\gamma_{d}}, \epsilon\right\}$ parameter space which has been converted into the purple region in the figure. Reference [10] also estimates that a future supernova ( $\mathrm{SN}$ ) explosion could produce visible gamma-ray fluxes for parameters within the purple dashed line.

Additional bounds come from the effects of late dark photon decays on primordial nucleosynthesis $[8,15]$. When $\gamma_{d}$ is heavier than two pions, decays into hadrons alter the $n / p$ ratio and destroy light elements, such as deuterium and helium. Measurements of $\mathrm{D} / \mathrm{H}$ and ${ }^{4} \mathrm{He}$ exclude the brown and the blue regions (these constraints are inferred from Ref. [15], imposing $m_{\gamma_{d}}>2 m_{\pi}$ ).

Finally, DHDM can have sizable self interactions, $h_{d} h_{d} \leftrightarrow h_{d} h_{d}$, with a cross section, $\sigma_{\mathrm{SIDM}} / m_{h_{d}}=$ $18 \pi \alpha_{d}^{2} m_{h_{d}} / m_{\gamma_{d}}^{4}$. The blue contours correspond to constant $\sigma_{\mathrm{SIDM}} / m_{h_{d}}$. In the upper left corner values of $1 \mathrm{~cm}^{2} / \mathrm{gr}$ can be achieved; of interest for astrophysical signals of DM [23].

\section{FREEZE-OUT}

For intermediate values of $\epsilon$, the DHDM relic density can be set by the freeze-out of secluded annihilations. Interestingly, this freeze-out scenario combines many properties which have previously been studied in Refs. [24-30]. The salient features can be summarized as follows. The DS is initially decoupled from the SM $\left(T_{d} \neq T_{\mathrm{SM}}\right)$, and has a mass gap and a small hierarchy with $m_{h_{d}} \lesssim m_{\gamma_{d}}$. Active number changing processes lead to a period of DS self-depletion and self-heating. Additional exponential depletion occurs through out-of-equilibrium decay of $\gamma_{d}$, leading to a delayed freeze-out of $h_{d} h_{d} \rightarrow$ $\gamma_{d} \gamma_{d}$, with a nonzero dark chemical potential.

The evolutions of $n_{h_{d}}, n_{\gamma_{d}}$, and $T_{d}$ follow from the same set of BEs (3)-(6) as before. Initial conditions are set by assuming that the DS is independently populated during reheating and subsequently reaches internal thermal equilibrium. At high temperatures, the SM and DS evolve as two decoupled baths and, for small $\epsilon$, they never equilibrate. Indeed, energy exchanging processes between the two sectors, dominated by $\gamma_{d} \leftrightarrow e^{+} e^{-}$at $T_{\mathrm{SM}} \approx m_{\gamma_{d}} \approx m_{h_{d}}$, remain slower than Hubble for $\epsilon \lesssim$ $10^{-8}\left(m_{\gamma_{d}} / 100 \mathrm{MeV}\right)^{1 / 2}$. For larger values of $\epsilon$, the standard secluded freeze-out scenario, with $T_{d}=T_{\mathrm{SM}}$, is recovered [31].

In the decoupled limit, if number changing processes remain active when $T_{d}<\min \left[m_{\gamma_{d}}, m_{h_{d}}\right]$, the DS maintains vanishing chemical potentials and undergoes a period of cannibalism, during which $T_{d}$ decreases logarithmically with the scale factor $[24,25]$. Cannibalism proceeds as long as $\left(n_{h_{d}}^{\mathrm{eq}}\right)^{2}\left\langle\sigma v^{2}\right\rangle_{h_{d} h_{d} h_{d} \rightarrow \gamma_{d} \gamma_{d}} \gtrsim H$ at $T_{d} \approx T_{\mathrm{SM}} \approx m_{h_{d}}$, which corresponds to $\alpha_{d} \gtrsim 10^{-6}\left(m_{h_{d}} / \mathrm{MeV}\right)^{1 / 3}$.

The dominant freeze-out annihilation channel, $h_{d} h_{d} \rightarrow$ $\gamma_{d} \gamma_{d}$, efficiently removes DHDM particles only when the decay process $\gamma_{d} \rightarrow e^{+} e^{-}$becomes active [26,27]. The latter also cools down the DS, inducing the approximate scaling $T_{d} \propto T_{\mathrm{SM}}^{2}$. Since $h_{d} h_{d} \rightarrow \gamma_{d} \gamma_{d}$ is kinematically forbidden at zero temperature, the rate becomes exponentially suppressed when $T_{d} \lesssim\left(m_{\gamma_{d}}-m_{h_{d}}\right)$. Therefore, the DHDM abundance is exponentially sensitive to the mass splitting and to the temperature at which $\gamma_{d}$ decays become active (see Appendix C for more details).

An example of the DS evolution showing the behavior described above is displayed in Fig. 6. We take $T_{\mathrm{SM}}=T_{d}$ at $1 \mathrm{TeV}$ and assume separate entropy conservation in the two sectors until $m_{h_{d}} / T_{\mathrm{SM}} \approx 4$ (vertical gray line in the figure). Below this temperature, the full set of BEs tracks the evolution of $n_{h_{d}}, n_{\gamma_{d}}$, and $T_{d}$. In the bottom panel, the departure of the green curve from the black marks the onset of $\gamma_{d}$ decay. In the top panel, the departure of the solid curves from the dashed corresponds to the freeze-out of $3 \leftrightarrow 2$ processes, with the DS particles developing a chemical potential.

\section{A. Phenomenological consequences}

The phenomenology of the secluded annihilation freezeout scenario is summarized in Fig. 7 for $\alpha_{d}=3 \times 10^{-3}$. At every point, $r$ is chosen to satisfy $\Omega_{h_{d}} h^{2}=0.12$. We find that for large $m_{\gamma_{d}}$ and small $\epsilon$, achieving the correct relic abundance requires a compressed spectrum.

Part of the parameter space is excluded by constraints on $\gamma_{d}$. The dark photon can induce excessive cooling of SN1987A [9] (see however Ref. [34]) or be excluded by the bound of Ref. [10], described above (orange and purple, respectively). Again, the parameter space with prospects for discovery of $\gamma_{d}$ by gamma-rays produced from a 


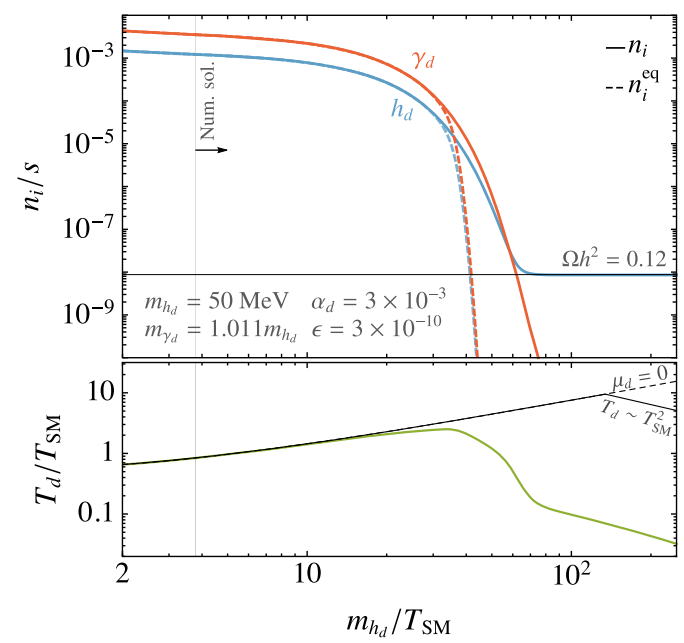

FIG. 6. Same as Fig. 5 for the secluded freeze-out regime. Separate entropy conservation in the two sectors is assumed to the left of the thin vertical line, while the full set of BEs (3)-(6) are solved to the right. The evolution of $T_{d}$ for a completely decoupled DS with a sudden freeze-out of $3 \rightarrow 2$ processes at $m_{h_{d}} / T_{\mathrm{SM}} \approx 130$ is shown as a black curve in the bottom panel.

future SN event is bounded by the dashed purple line. Additional constraints are specific to the DHDM scenario. Electromagnetic energy injection due to dark Higgs decays are constrained by measurements of $\mathrm{CMB}$ anisotropies $[6,7,35]$ (green). The long-standing SPI/INTEGRAL observation of a positron excess in the galactic bulge [32] could be explained by decaying DM $[33,36]$. Along the dashed black curve, DHDM can produce the observed photon flux, while in the gray region too many $e^{+}$are generated.

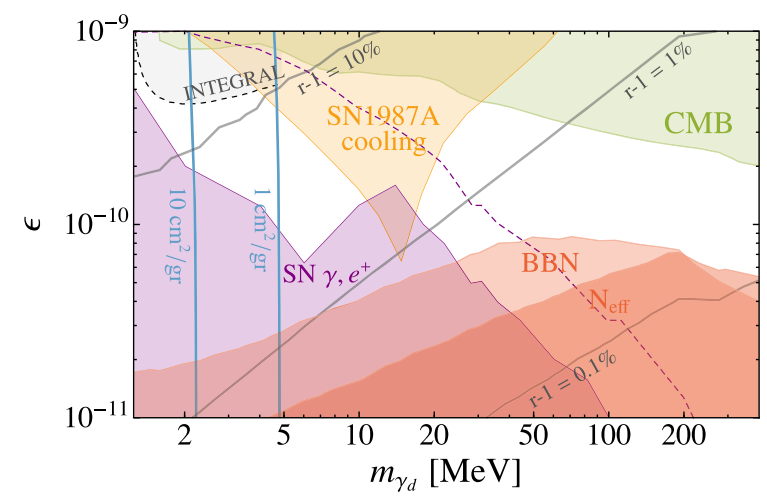

FIG. 7. Parameter space for the secluded annihilation freeze-out scenario for $\alpha_{d}=3 \times 10^{-3}$. The correct DHDM relic density is produced everywhere by fixing $r \equiv m_{\gamma_{d}} / m_{h_{d}}$; constant values are shown as gray contours. Bounds include dark photon constraints from SN1987A cooling [9], $e^{+}$and $\gamma$ signals from $\gamma_{d}$ produced within $\mathrm{SNe}$ [10] (discovery potential given by the purple dashed line), CMB anisotropies limits on late DHDM decay [6], and BBN and $N_{\text {eff }}$ [14] bounds on the fraction of dark energy density. Also shown are the region of parameter space that could explain the SPI/INTEGRAL anomaly [32,33], and blue contours of constant $\sigma_{\mathrm{SIDM}} / m_{h_{d}}$.
Small values of $\epsilon$ imply delayed $\gamma_{d}$ decays, leading to a prolonged period of cannibalism where the DS's energy density, $\rho_{d}$, dilutes less rapidly than the SM's, $\rho_{\mathrm{SM}}$. A sizable fraction of $\rho_{d}$ during the time of $\mathrm{BBN}(50 \mathrm{keV} \lesssim$ $T_{\mathrm{SM}} \lesssim 1 \mathrm{MeV}$ ) would increase Hubble and affect the observed primordial element abundances. We exclude parameters where $\rho_{d}>0.1 \rho_{\mathrm{SM}}$ at any time during BBN (light red). Moreover, if the additional $\rho_{d}$ is deposited into the photon bath through $\gamma_{d}$ decays after neutrino decoupling $\left(T_{\mathrm{SM}} \simeq 2 \mathrm{MeV}\right)$, the photon temperature is increased relative to neutrinos, lowering the value of $N_{\text {eff }}$ [37]. We show the $95 \%$ CL bound from Planck [14], $N_{\text {eff }}>2.55$ (dark red).

Finally, the freeze-out scenario also allows for sizable self-interaction cross sections $\sigma_{\mathrm{SIDM}} / m_{h_{d}}$, denoted by the blue curves.

\section{CONCLUSIONS}

This Article presents a minimal realization of the $U(1)_{d}$ model that includes a DM candidate: a cosmologically stable dark Higgs. We identify three distinct production mechanisms, which are nontrivial realizations of either freeze-in or freeze-out from the primordial plasma. The parameter space, $10^{-13} \lesssim \epsilon \lesssim 10^{-6}$ and $\mathrm{MeV} \lesssim m_{\gamma_{d}} \lesssim \mathrm{GeV}$, allows for viable DHDM with a rich cosmological history and diverse phenomenology. Detection of excess gammaray flux from a future SN explosion and/or astrophysical evidence of self interacting DM offer experimental probes of the model.

\section{ACKNOWLEDGMENTS}

We thank H. Liu, P. Meade, A. Pierce, and H. Ramani for helpful discussions. J. T. R. is supported by NSF CAREER Grant No. PHY-1554858 and NSF Grant No. PHY1915409. J. T. R. acknowledges hospitality from the Aspen Center for Physics, which is supported by the NSF Grant No. PHY-1607611. C. M. is partially supported by Perimeter Institute for Theoretical Physics. Research at Perimeter Institute is supported in part by the Government of Canada through the Department of Innovation, Science and Economic Development Canada and by the Province of Ontario through the Ministry of Economic Development, Job Creation and Trade.

\section{APPENDIX A: BOLTZMANN EQUATIONS FOR A DARK SECTOR}

The microscopic evolution of an arbitrary DS particle's phase space distribution $f_{\psi}$ can be traced to the $\operatorname{BE} \hat{L}\left[f_{\psi}\right]=$ $C\left[f_{\psi}\right]$ [38], where $\hat{L}$ is the Liouville operator and $C\left[f_{\psi}\right]$ is the collision operator which involves particle species $\psi$. In a Friedmann-Robertson-Walker Universe the former reads $\hat{L}\left[f_{\psi}\right] \equiv E_{\psi} \dot{f}_{\psi}-H \mathbf{p}_{\psi}^{2} \partial_{E_{\psi}} f_{\psi}$, where $E_{\psi}$ and $\mathbf{p}_{\psi}$ are the energy and momentum of $\psi$ particles. The $n$th moment of the BE is 


$$
\frac{g_{\psi}}{(2 \pi)^{3}} \int \frac{\mathrm{d} \mathbf{p}_{\psi}}{E_{\psi}} E_{\psi}^{n} \hat{L}\left[f_{\psi}\right]=\frac{g_{\psi}}{(2 \pi)^{3}} \int \frac{\mathrm{d} \mathbf{p}_{\psi}}{E_{\psi}} E_{\psi}^{n} C\left[f_{\psi}\right]
$$

The 0th and 1st moments can be written in terms of $\psi$ number density $n_{\psi}$, energy density $\rho_{\psi}$, and pressure $P_{\psi}$ as

$$
\begin{gathered}
\dot{n}_{\psi}+3 H n_{\psi}=\frac{g_{\psi}}{(2 \pi)^{3}} \int \frac{\mathrm{d} \mathbf{p}_{\psi}}{E_{\psi}} C\left[f_{\psi}\right], \\
\dot{\rho}_{\psi}+3 H\left(\rho_{\psi}+P_{\psi}\right)=\frac{g_{\psi}}{(2 \pi)^{3}} \int \frac{\mathrm{d} \mathbf{p}_{\psi}}{E_{\psi}} E_{\psi} C\left[f_{\psi}\right] .
\end{gathered}
$$

Assuming that $\psi$ maintains kinetic equilibrium with the DS bath, the 1st moment of the BE tracks the evolution of the dark temperature, $T_{d}$. Note that deviations from equilibrium would require solving for higher moments of the BEs; any such higher order effects have been neglected in this study.

In what follows we develop a number of useful equations for the 0th and 1st moments of the BEs. We begin with a nonrelativistic treatment which has the benefit of providing intuition regarding the behavior of the equations. We follow with a full relativistic treatment for completeness. Calculations presented in this study have been performed using the full relativistic equations when the result is sensitive to such corrections.

\section{Nonrelativistic regime}

In the nonrelativistic regime, at leading order in $T_{d} / m_{\psi}$ and $p_{\psi} / m_{\psi}$ (where $p_{\psi} \equiv\left|\mathbf{p}_{\psi}\right|$ is the three-momentum), one can make the following replacements in Eq. (A3):

$$
\begin{aligned}
& P_{\psi} \approx n_{\psi} T_{d}, \quad \rho_{\psi} \approx n_{\psi} m_{\psi}\left(1+\frac{3}{2} \frac{T_{d}}{m_{\psi}}\right), \\
& E_{\psi} \approx m_{\psi}+\frac{\mathbf{p}_{\psi}^{2}}{2 m_{\psi}},
\end{aligned}
$$

to obtain

$$
\begin{gathered}
\left(m_{\psi}+\frac{3}{2} T_{d}\right)\left(\dot{n}_{\psi}+3 H n_{\psi}\right)+\frac{3}{2} n_{\psi} T_{d}\left(\frac{\dot{T}_{d}}{T_{d}}+2 H\right) \\
\approx \frac{g_{\psi}}{(2 \pi)^{3}} \int \frac{\mathrm{d} \mathbf{p}_{\psi}}{E_{\psi}}\left(m_{\psi}+\frac{\mathbf{p}_{\psi}^{2}}{2 m_{\psi}}\right) C\left[f_{\psi}\right] .
\end{gathered}
$$

Note that the 0th order term in the nonrelativistic expansion cancels out by inserting Eq. (A2) into Eq. (A5). Explicitly writing the collision term for a general process, $j$, that involves $\psi$ particles, the result is,

$$
\begin{aligned}
& \frac{3}{2} T_{d}\left(\dot{n}_{\psi}+3 H n_{\psi}\right)+\frac{3}{2} n_{\psi} T_{d}\left(\frac{\dot{T}_{d}}{T_{d}}+2 H\right) \\
& \quad \approx \int d \Pi \delta^{4}\left(p_{\text {fin }}-p_{\text {init }}\right)|\mathcal{M}|_{j}^{2}\left[\prod f_{\text {fin }}-\prod f_{\text {init }}\right] \frac{\mathbf{p}_{\psi}^{2}}{2 m_{\psi}},
\end{aligned}
$$

where $p_{\text {init }}$ and $p_{\text {fin }}$ are the total initial and final state momenta, respectively, $\delta(\ldots) \equiv 2 \pi \delta(\ldots), \quad d \Pi=\prod_{i} g_{i} /$ $(2 \pi)^{3} \mathrm{~d} \mathbf{p}_{i} /\left(2 E_{i}\right)$ corresponds to the phase space integral over all incoming and outgoing particles, $|\mathcal{M}|_{j}^{2}$ is the matrix element squared averaged over initial and final spins (including symmetry factors for initial and final identical particles), and $\prod f_{\text {init }}$ and $\prod f_{\text {fin }}$ are products of the initial and final distribution functions, respectively. In this study, Maxwell-Boltzmann statistics are always assumed. In Eq. (A6), the terms proportional to $m_{\psi}$ have canceled out. The collision term on the right-hand side of the equation should include all energy exchanging processes involving $\psi$. This term has a particularly simple expression for decay processes of the form $\psi \rightarrow$ SM SM,

$\dot{n}_{\psi}+3 H n_{\psi}+n_{\psi}\left(\frac{\dot{T}_{d}}{T_{d}}+2 H\right)=-\Gamma_{\psi}^{0}\left[n_{\psi}-\frac{T_{\mathrm{SM}}}{T_{d}} n_{\psi}^{\mathrm{eq}}(T)\right]$.

Here, $\Gamma_{\psi}^{0}$ is the zero temperature decay width.

If all the particles in the DS are nonrelativistic and maintain kinetic equilibrium with each other throughout, summing over Eq. (A3) [or equivalently Eq. (A5)] for all dark $\psi$ particles, results in a collision term that includes only interactions between the DS and the SM. If these interactions can be neglected, the collision term vanishes and the total DS energy is conserved,

$$
\begin{aligned}
& \sum_{\psi}\left[\left(m_{\psi}+\frac{3}{2} T_{d}\right)\left(\dot{n}_{\psi}+3 H n_{\psi}\right)+\frac{3}{2} T_{d} n_{\psi}\left(\frac{\dot{T}_{d}}{T_{d}}+2 H\right)\right] \\
& \quad=0,
\end{aligned}
$$

where, as stated, the sum is taken over all DS particles. From the above equation, the effect on the evolution of $T_{d}$ of mass conversion into kinetic energy, and, vice versa, becomes evident by replacing $\dot{n}_{\psi}+3 H n_{\psi}$ with the righthand side of Eq. (A2).

For completeness, the full set of BEs for the model discussed in the main text are given below. The 0th moments of the BEs for the two species in the DS, including all relevant terms, are

$$
\begin{aligned}
\dot{n}_{h_{d}}+3 H n_{h_{d}}= & \mathcal{C}_{2 \gamma_{d} \rightarrow 2 h_{d}}-\mathcal{C}_{3 h_{d} \rightarrow 2 \gamma_{d}}-\mathcal{C}_{3 h_{d} \rightarrow 2 h_{d}} \\
& +-\mathcal{C}_{2 h_{d} \gamma_{d} \rightarrow h_{d} \gamma_{d}}, \\
\dot{n}_{\gamma_{d}}+3 H n_{\gamma_{d}}= & -\mathcal{C}_{\gamma_{d} \rightarrow e^{+} e^{-}}-\mathcal{C}_{2 \gamma_{d} \rightarrow 2 h_{d}}+\frac{2}{3} \mathcal{C}_{3 h_{d} \rightarrow 2 \gamma_{d}},
\end{aligned}
$$

with collision terms 


$$
\begin{aligned}
\mathcal{C}_{\gamma_{d} \rightarrow e^{+} e^{-}} & \equiv \Gamma_{\gamma_{d}}^{0}\left[\frac{K_{1}\left(r x_{d}\right)}{K_{2}\left(r x_{d}\right)} n_{\gamma_{d}}-\frac{K_{1}(r x)}{K_{2}(r x)} n_{\gamma_{d}}^{\mathrm{eq}}\left(T_{\mathrm{SM}}\right)\right], \\
\mathcal{C}_{2 \gamma_{d} \rightarrow 2 h_{d}} & \equiv\langle\sigma v\rangle_{\gamma_{d} \gamma_{d} \rightarrow h_{d} h_{d}}\left[n_{\gamma_{d}}^{2}-\frac{n_{\gamma_{d}}^{\mathrm{eq} 2}}{n_{h_{d}}^{\mathrm{eq} 2}} n_{h_{d}}^{2}\right], \\
\mathcal{C}_{3 h_{d} \rightarrow 2 \gamma_{d}} & \equiv \frac{3}{3 ! 2 !}\left\langle\sigma v^{2}\right\rangle_{h_{d} h_{d} h_{d} \rightarrow \gamma_{d} \gamma_{d}}\left[n_{h_{d}}^{3}-\frac{n_{h_{d}}^{\mathrm{eq} 3}}{n_{\gamma_{d}}^{\mathrm{eq} 2}} n_{\gamma_{d}}^{2}\right], \\
\mathcal{C}_{3 h_{d} \rightarrow 2 h_{d}} & \equiv \frac{1}{3 ! 2 !}\left\langle\sigma v^{2}\right\rangle_{h_{d} h_{d} h_{d} \rightarrow h_{d} h_{d}}\left[n_{h_{d}}^{3}-n_{h_{d}}^{\mathrm{eq}} n_{h_{d}}^{2}\right], \\
\mathcal{C}_{2 h_{d} \gamma_{d} \rightarrow h_{d} \gamma_{d}} & \equiv \frac{1}{2 !}\left\langle\sigma v^{2}\right\rangle_{h_{d} h_{d} \gamma_{d} \rightarrow h_{d} \gamma_{d}}\left[n_{h_{d}}^{2} n_{\gamma_{d}}-n_{h_{d}}^{\mathrm{eq}} n_{h_{d}} n_{\gamma_{d}}\right] .
\end{aligned}
$$

Here, $n_{i}^{\mathrm{eq}} \equiv n_{i}^{\mathrm{eq}}\left(T_{d}\right)$ is the equilibrium number density (with zero chemical potential) of particle $i=h_{d}, \gamma_{d}$ as a function of the hidden sector temperature, while $n_{i}^{\mathrm{eq}}\left(T_{\mathrm{SM}}\right)$ is evaluated at the $\mathrm{SM}$ temperature. $K_{1 / 2}$ are modified Bessel functions of the 2nd kind, $x_{d} \equiv m_{h_{d}} / T_{d}$, $x \equiv m_{h_{d}} / T_{\mathrm{SM}}, r=m_{\gamma_{d}} / m_{h_{d}}$, and $\langle\sigma v\rangle$ and $\left\langle\sigma v^{2}\right\rangle$ denote the thermal averaged cross sections for $2 \rightarrow 2$ and $3 \rightarrow 2$ processes, respectively. The cross sections for $2 \rightarrow 3$ processes are obtained by computing $\sigma_{2 \rightarrow 3}$ numerically with CalcHEP [39] and integrating over the center of mass energy to get the thermal average at different temperatures using the usual formula from Ref. [40] adapted to $2 \rightarrow 3$ processes. The expressions for the inverse processes are obtained by applying detailed balance.

Defining $n=n_{h_{d}}+n_{\gamma_{d}}$, the dark temperature evolution equation in the nonrelativistic regime is

$$
\begin{aligned}
n \frac{\dot{T_{d}}}{T_{d}}+2 H n= & -\mathcal{C}_{\gamma_{d} \rightarrow e^{+} e^{-}}^{(E), \mathrm{nr}}+\mathcal{C}_{2 \gamma_{d} \rightarrow 2 h_{d}}^{(E), \mathrm{nr}}+\frac{2}{3} \mathcal{C}_{3 h_{d} \rightarrow 2 \gamma_{d}}^{(E), \mathrm{nr}} \\
& +\mathcal{C}_{3 h_{d} \rightarrow 2 h_{d}}^{(E), \mathrm{nr}}+\mathcal{C}_{2 h_{d \gamma_{d}} \rightarrow h_{d} \gamma_{d}}^{(E), \mathrm{rr}}
\end{aligned}
$$

with collision terms

$$
\begin{aligned}
& \mathcal{C}_{\gamma_{d} \rightarrow e^{+} e^{-}}^{(E), \mathrm{nr}} \equiv \Gamma_{\gamma_{d}}^{0}\left[\left(1-\frac{K_{1}\left(r x_{d}\right)}{K_{2}\left(r x_{d}\right)}\right) n_{\gamma_{d}}\right. \\
&\left.+\left(\frac{K_{1}(r x)}{K_{2}(r x)}-\frac{T_{\mathrm{SM}}}{T_{d}}\right) n_{\gamma_{d}}^{\mathrm{eq}}(T)\right], \\
& \mathcal{C}_{2 \gamma_{d} \rightarrow 2 h_{d}}^{(E), \mathrm{nr}} \equiv \frac{2}{3}(r-1) x_{d} \mathcal{C}_{2 \gamma_{d} \rightarrow 2 h_{d}}, \\
& \mathcal{C}_{3 h_{d} \rightarrow 2 \gamma_{d}}^{(E), \mathrm{nr}} \equiv {\left[\left(1-\frac{2}{3} r\right) x_{d}+\frac{1}{2}\right] \mathcal{C}_{3 h_{d} \rightarrow 2 \gamma_{d}}, } \\
& \mathcal{C}_{3 h_{d} \rightarrow 2 h_{d}}^{(E), \mathrm{nr}} \equiv\left(\frac{2}{3} x_{d}+1\right) \mathcal{C}_{3 h_{d} \rightarrow 2 h_{d}}, \\
& \mathcal{C}_{2 h_{d \gamma_{d} \rightarrow h_{d} \gamma_{d}}^{(E), \mathrm{nr}} \equiv}\left(\frac{2}{3} x_{d}+1\right) \mathcal{C}_{2 h_{d} \gamma_{d} \rightarrow h_{d} \gamma_{d}} .
\end{aligned}
$$

Note that Eq. (A11) is only valid in the nonrelativistic regime and therefore one need only consider leading terms in the Bessel functions within $\mathcal{C}_{\gamma_{d} \rightarrow e^{+} e^{-}}^{(E), n}$. We have verified that the processes $h_{d} \gamma_{d} \leftrightarrow e^{+} e^{-}, \quad \gamma_{d} e^{ \pm} \leftrightarrow \gamma e^{ \pm}$, and $h_{d} e^{ \pm} \leftrightarrow \gamma_{d} e^{ \pm}$are negligible. As expected for a nonrelativistic mediator, $2 \leftrightarrow 2$ processes are subdominant with respect to $1 \leftrightarrow 2$, due to the extra insertion of electromagnetic or dark gauge couplings. In the scenarios considered in this work, the Hubble parameter is always dominated by the SM energy density, but regardless the contribution from the DS is included in the numerical results.

\section{General treatment with classical statistics}

For arbitrary values of the temperature, the $\mathrm{BE}$ for the energy density, Eq. (A3), can still be converted into a dark sector temperature evolution equation for particles with Maxwell-Boltzmann distributions. The result is a generalized and slightly more complex version of Eq. (A6) that is valid, in the limit of classical statistics, even at temperatures above the masses of the particles involved. For notational conciseness, the evolution equation is written for the variable $x_{d} \equiv m_{\psi} / T_{d}$.

The number density, pressure, and energy density for the species $\psi$ can be explicitly written as

$n_{\psi}=\frac{g_{\psi}}{2 \pi^{2}} \frac{m_{\psi}^{3}}{x_{d}} K_{2}\left(x_{d}\right) e^{\mu_{\psi} / T_{d}}, \quad P_{\psi}=\frac{m_{\psi} n_{\psi}}{x_{d}}$,

$\rho_{\psi}=m_{\psi} n_{\psi}\left[\frac{K_{1}\left(x_{d}\right)}{K_{2}\left(x_{d}\right)}+\frac{3}{x_{d}}\right] \equiv m_{\psi} n_{\psi} F\left(x_{d}\right)$,

where $K_{1 / 2}$ are modified Bessel functions of the 2nd kind, $g_{\psi}$ denotes $\psi$ internal degrees of freedom, $\mu_{\psi}$ is the chemical potential, and we have introduced the function $F\left(x_{d}\right)$. The time derivative of the energy density is then

$$
\dot{\rho}_{\psi}=m_{\psi} \dot{n}_{\psi} F\left(x_{d}\right)+m_{\psi} n_{\psi} F^{\prime}\left(x_{d}\right) \dot{x}_{d},
$$

where the dot operator denotes a derivative with respect to time and the prime operator denotes a derivative with respect to $x_{d}$. Equation (A3) becomes

$$
\begin{aligned}
m_{\psi} & F\left(x_{d}\right)\left(\dot{n}_{\psi}+3 H n_{\psi}\right)+\frac{m_{\psi} n_{\psi}}{x_{d}}\left(x_{d} F^{\prime}\left(x_{d}\right) \dot{x}_{d}+3 H\right) \\
& =\frac{g_{\psi}}{(2 \pi)^{3}} \int \frac{\mathrm{d} \mathbf{p}_{\psi}}{E_{\psi}} E_{\psi} C\left[f_{\psi}\right] .
\end{aligned}
$$

If the dominant energy exchange processes for the particle $\psi$ are its decay and inverse decay $\psi \leftrightarrow$ SM SM, the relativistic analog of Eq. (A7) is

$$
\begin{aligned}
& F\left(x_{d}\right)\left(\dot{n}_{\psi}+3 H n_{\psi}\right)+\frac{n_{\psi}}{x_{d}}\left(x_{d} F^{\prime}\left(x_{d}\right) \dot{x}_{d}+3 H\right) \\
& \quad=-\Gamma_{\psi}^{0}\left[n_{\psi}-n_{\psi}^{\mathrm{eq}}\left(T_{\mathrm{SM}}\right)\right] .
\end{aligned}
$$

The nonrelativistic approximation is recovered by explicitly writing the $\mathrm{BE}$ for the $\psi$ number density [Eq. (A2)], 
keeping only the $\psi \leftrightarrow$ SM SM collision term, and expanding Bessel functions and $F\left(x_{d}\right)$ to first order for large $x_{d}$ and $x$.

The analog of Eq. (A8), obtained by summing over all DS particles $\psi$, for the case of a DS in internal kinetic equilibrium and negligible interactions with the SM, is

$$
\sum_{\psi}\left[x_{d} F\left(x_{d}\right)\left(\dot{n}_{\psi}+3 H n_{\psi}\right)+n_{\psi}\left(x_{d} F^{\prime}\left(x_{d}\right) \dot{x}_{d}+3 H\right)\right]=0 .
$$

The effects of mass conversion into kinetic energy, and vice versa, on the evolution of $T_{d}$ becomes evident by replacing $\dot{n}_{\psi}+3 H n_{\psi}$ with the right-hand side of Eq. (A2).

For completeness, the dark temperature evolution equation for the model discussed in the main text is

$$
\begin{aligned}
& {\left[n_{h_{d}} F^{\prime}\left(x_{d}\right)+r^{2} n_{\gamma_{d}} F^{\prime}\left(r x_{d}\right)\right] \dot{x}_{d}+\frac{3 H n}{x_{d}}} \\
& =-\mathcal{C}_{\gamma_{d} \rightarrow e^{+} e^{-}}^{(E)}+\mathcal{C}_{2 \gamma_{d} \rightarrow 2 h_{d}}^{(E)}+\mathcal{C}_{3 h_{d} \rightarrow 2 \gamma_{d}}^{(E)} \\
& \quad+\mathcal{C}_{3 h_{d} \rightarrow 2 h_{d}}^{(E)}+\mathcal{C}_{2 h_{d} \gamma_{d} \rightarrow h_{d} \gamma_{d}}^{(E)},
\end{aligned}
$$

where $x_{d} \equiv m_{h_{d}} / T_{d}, \quad x \equiv m_{h_{d}} / T_{\mathrm{SM}}, \quad r \equiv m_{\gamma_{d}} / m_{h_{d}}, \quad$ and $n \equiv n_{h_{d}}+n_{\gamma_{d}}$. The collision terms are redefined as

$$
\begin{aligned}
\mathcal{C}_{\gamma_{d} \rightarrow e^{+} e^{-}}^{(E)} \equiv & r \Gamma_{\gamma_{d}}^{0}\left\{\left[1-F\left(r x_{d}\right) \frac{K_{1}\left(r x_{d}\right)}{K_{2}\left(r x_{d}\right)}\right] n_{\gamma_{d}}\right. \\
& \left.+-\left[1-F\left(r x_{d}\right) \frac{K_{1}(r x)}{K_{2}(r x)}\right] n_{\gamma_{d}}^{\mathrm{eq}}\left(T_{\mathrm{SM}}\right)\right\} \\
\mathcal{C}_{2 \gamma_{d} \rightarrow 2 h_{d}}^{(E)} \equiv & {\left[r F\left(r x_{d}\right)-F\left(x_{d}\right)\right] \mathcal{C}_{2 \gamma_{d} \rightarrow 2 h_{d}}, } \\
\mathcal{C}_{3 h_{d} \rightarrow 2 \gamma_{d}}^{(E)} \equiv & {\left[F\left(x_{d}\right)-\frac{2}{3} r F\left(r x_{d}\right)\right] \mathcal{C}_{3 h_{d} \rightarrow 2 \gamma_{d}}, } \\
\mathcal{C}_{3 h_{d} \rightarrow 2 h_{d}}^{(E)} \equiv & F\left(x_{d}\right) \mathcal{C}_{3 h_{d} \rightarrow 2 h_{d}}, \\
\mathcal{C}_{2 h_{d} \gamma_{d} \rightarrow h_{d} \gamma_{d}}^{(E)} \equiv & F\left(x_{d}\right) \mathcal{C}_{2 h_{d} \gamma_{d} \rightarrow h_{d} \gamma_{d}}
\end{aligned}
$$

where the number changing collision terms are equivalent to those in Eqs. (A10).

\section{APPENDIX B: REPRODUCTIVE FREEZE-IN}

The 0th moment of the BE, Eq. (A2), can be written in the general form,

$$
\begin{aligned}
\dot{n}_{\psi}+3 H n_{\psi} & =\sum_{j} \frac{N_{\psi, j}}{S_{j}} \mathcal{C}_{\text {init } \rightarrow \mathrm{fin}, j} \\
& \equiv \sum_{j} \frac{N_{\psi, j}}{S_{j}} n_{\text {init }, j}^{\mathrm{eq}}\langle\sigma v\rangle_{\mathrm{init} \rightarrow \mathrm{fin}, j}\left(\frac{n_{\mathrm{init}, j}}{n_{\mathrm{init}, j}^{\mathrm{eq}}}-\frac{n_{\mathrm{fin}, j}}{n_{\mathrm{fin}, j}^{\mathrm{eq}}}\right),
\end{aligned}
$$

where the sum is over all relevant processes of the form init $\rightarrow$ fin, which change the number of $\psi$ particles (indexed by subscript $j$ ). $\quad N_{\psi, j} \equiv N_{\psi, \text { fin }, j}-N_{\psi, \text { init }, j}$ is defined as the difference between the number of outgoing and incoming $\psi$ particles in process $j . S_{j}$ is a combinatorial factor which counts the number of ways to sort all initial and final particles in process $j$. Finally, $n_{\text {init/fin, } j}^{(\mathrm{eq})}$ are products of (equilibrium) number densities of all particles in the initial or final state of process $j$.

For the case where all particles in the interaction $j$ belong to the DS, one can plug Eq. (B1) into Eq. (A17), which results in

$$
\sum_{\psi} n_{\psi}\left[x_{d} F^{\prime}\left(x_{d}\right) \dot{x}_{d}+3 H\right]=-\sum_{j} \sum_{\psi} \frac{N_{\psi, j}}{S_{j}} x_{d} F\left(x_{d}\right) \mathcal{C}_{\mathrm{init} \rightarrow \mathrm{fin}, j},
$$

or, in the nonrelativistic limit,

$$
\sum_{\psi} n_{\psi}\left(\frac{\dot{T}_{d}}{T_{d}}+2 H\right)=-\sum_{j} \sum_{\psi} \frac{N_{\psi, j}}{S_{j}}\left(\frac{2}{3} \frac{m_{\psi}}{T_{d}}+1\right) \mathcal{C}_{\text {init } \rightarrow \text { fin }, j} .
$$

The dark sector could be initially populated at relativistic values of $T_{d}$, therefore requiring the solution of the full Eq. (B2). However, as shown below, reproductive freeze-in always results in a $T_{d}$ evolution that is independent of initial conditions. Thus, the solutions of Eqs. (B2) and (B3) lead to comparable results.

Consider a DS which consists of two species $A$ and $B$, as is the case in the current study. As described in the main text, the reproductive regime occurs when $2 \leftrightarrow 2$ as well as $2 \rightarrow 3$ interactions involving DS particles only are simultaneously faster than the Hubble rate. In this case, the $2 \leftrightarrow 2$ interactions, $A A \leftrightarrow B B, A A \leftrightarrow A B$, and $B B \leftrightarrow A B$ enforce chemical equilibrium between $A$ and $B$, namely,

$$
\frac{n_{A}}{n_{A}^{\mathrm{eq}}} \approx \frac{n_{B}}{n_{B}^{\mathrm{eq}}}
$$

The collision term for any $3 \leftrightarrow 2$ interaction, $j$, within the DS can then be found by plugging Eq. (B4) into the definition of $\mathcal{C}_{\text {init } \rightarrow \text { fin, }, j}$ for such a process, Eq. (B1). The result is

$$
\mathcal{C}_{2 \rightarrow 3, j}=n_{2, j}\langle\sigma v\rangle_{2 \rightarrow 3, j}\left(\frac{n_{A / B}}{n_{A / B}^{\mathrm{eq}}}-1\right) \approx-n_{2, j}\langle\sigma v\rangle_{2 \rightarrow 3, j},
$$

where the second equality is valid for the case where $n_{A / B} \ll n_{A / B}^{\mathrm{eq}}$, as is typically the case for freeze-in. Thus, the reaction always proceeds only in the $2 \rightarrow 3$ direction 
until the inequality no longer holds, rapidly forcing the dark temperature to a value for which the chemical potentials for both $A$ and $B$ vanish, i.e., $n_{A} \approx n_{A}^{\mathrm{eq}}$ and $n_{B} \approx n_{B}^{\mathrm{eq}}$. Importantly, this mechanism essentially wipes out all initial conditions of the dark temperature.

The rapid cooling described above can be seen explicitly by plugging Eq. (B5) into the temperature evolution Eq. (B2). Denoting $x_{d} \equiv m_{A} / T_{d}, r \equiv m_{B} / m_{A}$ and $F_{A / B}\left(m_{A / B} / T_{d}\right) \equiv F_{A / B}$ (suppressing the explicit dependence on $m_{A / B} / T_{d}$ ), one obtains

$$
\begin{aligned}
& \left(n_{A} F_{A}^{\prime}+n_{B} r^{2} F_{B}^{\prime}\right) x_{d} \dot{x}_{d}+\left(n_{A}+n_{B}\right) 3 H \\
& =\sum_{j} x_{d}\left[N_{B, j} r F_{B}-N_{A, j} F_{A}\right] \frac{\mathcal{C}_{2 \rightarrow 3, j}}{S_{j}},
\end{aligned}
$$

or, in the nonrelativistic limit,

$$
\left(n_{A}+n_{B}\right)\left(\frac{\dot{T}_{d}}{T_{d}}+2 H\right)=\sum_{j}\left[\frac{2}{3} \frac{M_{3, j}-M_{2, j}}{T_{d}}+1\right] \frac{\mathcal{C}_{2 \rightarrow 3, j}}{S_{j}},
$$

where $M_{2, j}\left(M_{3, j}\right)$ is the sum of masses of incoming (outgoing) particles for the $2 \rightarrow 3$ interaction. Note that in the reproductive regime $\mathcal{C}_{2 \rightarrow 3, j}<0$, and therefore $M_{3, j}>M_{2, j}$ corresponds to a cooling term as should be expected, since this corresponds to a transfer of kinetic energy to rest mass. Even when $M_{2, j}=M_{3, j}$, the constant term within the parentheses still enforces cooling. This is the result of removal of kinetic energy corresponding to the growth in number of particles in a $2 \rightarrow 3$ interaction. More generally, any number changing process, $j$, will induce a temperature drop when the inequality,

$$
N_{B, j} r F_{B}-N_{A, j} F_{A}>0,
$$

is satisfied. In the nonrelativistic limit, the above inequality becomes

$$
\frac{M_{3, j}-M_{2, j}}{T_{d}}>-\frac{3}{2}
$$

Such cooling can be more rapid than Hubble cooling if,

$$
\left|\frac{x_{d}}{S_{j}}\left[N_{B, j} r F_{B}-N_{A, j} F_{A}\right] \frac{n_{2, j}\langle\sigma v\rangle_{2 \rightarrow 3, j}}{n_{A}+n_{B}}\right|>3 H,
$$

or, in the nonrelativistic limit,

$$
\left|\frac{1}{S_{j}}\left[\frac{2}{3} \frac{M_{3, j}-M_{2, j}}{T_{d}}+1\right] \frac{n_{2, j}\langle\sigma v\rangle_{2 \rightarrow 3, j}}{n_{A}+n_{B}}\right|>2 H .
$$

\section{APPENDIX C: FREEZE-OUT OF SECLUDED ANNIHILATIONS}

As described in the main text, the secluded annihilation freeze-out scenario considered in this study shares various features with previous studies in the literature. The general picture can be understood as follows.

(i) The small coupling to the SM does not allow for full thermalization between the two sectors and thus the entire process described below occurs with a nontrivial evolution of the dark temperature.

(ii) The DS features sizable $3 \leftrightarrow 2$ interactions and, thus, at intermediate temperatures, undergoes a period of cannibalism. When $T_{d}$ drops below min $\left[m_{h_{d}}, m_{\gamma_{d}}\right]$, this results in a dark temperature which scales logarithmically with the SM temperature.

(iii) The depletion of DS particles occurs through the decay of dark photons via $\gamma_{d} \rightarrow e^{+} e^{-}$, delaying the freeze-out of secluded annihilation, $h_{d} h_{d} \rightarrow \gamma_{d} \gamma_{d}$, to $x \gg 20$.

(iv) The mass hierarchy considered in this study is $m_{h_{d}}<m_{\gamma_{d}}$, which corresponds to a DM annihilation process that is kinematically forbidden at zero temperature. This significantly alters freeze-out with respect to the cases of an inverted or degenerate mass spectrum.

Clearly, the entire evolution is captured within the numerical solutions of the BEs (3)-(6) in the main text. However, much of the qualitative behavior of a DS in such a regime can be understood analytically. The goal of this Appendix is to provide the relevant equations, together with an intuitive understanding of this evolution.

The initial conditions for the evolution are a DS which is completely decoupled from the SM, with some initial DS to SM entropy ratio, $\xi_{s} \equiv s_{d} / s_{\mathrm{SM}}$. Using the SM temperature, $T_{\mathrm{SM}}$, as an independent variable, the dark temperature, $T_{d}\left(T_{\mathrm{SM}}\right)$, can be obtained by requiring separate conservation of entropy in each sector. The low temperature behavior is particularly informative. When $T_{d}$ drops below $\min \left[m_{h_{d}}, m_{\gamma_{d}}\right]$, if number changing processes remain active, the DS's chemical potential is forced to zero. For $m_{h_{d}} / T_{d} \gg 5 / 2$, the total hidden sector entropy density is

$$
s_{d} \approx \frac{m_{h_{d}} n_{h_{d}}^{\mathrm{eq}}+m_{\gamma_{d}} n_{\gamma_{d}}^{\mathrm{eq}}}{T_{d}} .
$$

Using separate entropy conservation in each sector, one can directly solve for $x_{d} \equiv m_{h_{d}} / T_{d}$ as a function of $x \equiv m_{h_{d}} / T_{\mathrm{SM}}$,

$$
x_{d} \approx \ln \left(\frac{x^{3}}{1.7 \xi_{s} g_{*, s}}\right),
$$

which is the logarithmic dependence typical of a cannibalizing sector [24,25]. Neglecting possible energy transfer to or from the SM, this equation describes the evolution of 
$T_{d}$ until number changing processes within the DS become slower than the Hubble rate. From that point onwards, the dark temperature evolves approximately as $x_{d} \propto x^{2}$. Note that, depending on the size of the kinetic mixing parameter, energy transfer between the DS and the SM via the process $\gamma_{d} \leftrightarrow e^{+} e^{-}$might not be completely negligible. However, the above result serves as a reasonable approximation in the limit of small portal coupling.

Simultaneously, the annihilation process $h_{d} h_{d} \leftrightarrow \gamma_{d} \gamma_{d}$, forces chemical equilibrium such that $n_{h_{d}} / n_{h_{d}}^{\mathrm{eq}}=n_{\gamma_{d}} / n_{\gamma_{d}}^{\mathrm{eq}}$, i.e., the dark particles develop nonvanishing and equal chemical potentials. One can now use the requirement of chemical equilibrium together with the rough dark temperature evolution described above to approximately determine the SM (DS) temperature, $x^{\Gamma}\left(x_{d}^{\Gamma}\right)$, at which $\gamma_{d}$ begins decaying. This $x^{\Gamma}$ can be found by requiring that

$$
\frac{n_{\gamma_{d}}^{\mathrm{eq}}\left(x_{d}^{\Gamma}\right)}{n_{h_{d}}^{\mathrm{eq}}\left(x_{d}^{\Gamma}\right)+n_{\gamma_{d}}^{\mathrm{eq}}\left(x_{d}^{\Gamma}\right)} \Gamma_{\gamma_{d}}^{0}=H\left(x^{\Gamma}\right) .
$$

During the period where the decay process is active, and as long as $h_{d} h_{d} \leftrightarrow \gamma_{d} \gamma_{d}$ exchanges remain efficient, depletion of the DHDM number density proceeds indirectly through the decay channel, since the $\gamma_{d}$ particles become exponentially less abundant as the temperature drops. To gain intuition regarding the evolution of the DS, it is insightful to solve an approximate BE in the limit where the two sectors are completely decoupled and cannibalism turns off abruptly at $x^{\Gamma}$. In this case, the temperatures of the two sectors relate via $x_{d} \approx R_{\Gamma} x^{2}$, where $R_{\Gamma}$ is set by the initial condition at approximately $x^{\Gamma}$. Then, as long as the inverse decay process can be neglected, the total number density of DS particles, $n=n_{h_{d}}+n_{\gamma_{d}}$, can be approximated by

$$
\dot{n}+3 H n \approx-\frac{n_{\gamma_{d}}^{\mathrm{eq}}}{n^{\mathrm{eq}}} n \Gamma_{\gamma_{d}}^{0} \approx \frac{n}{s} \frac{x}{1+\frac{1}{3 r^{3 / 2}} e^{\Delta R_{\Gamma} x^{2}}} \frac{\Gamma_{\gamma_{d}}^{0}}{H_{m}},
$$

where $\quad r \equiv m_{\gamma_{d}} / m_{h_{d}}, \quad \Delta \equiv r-1, \quad$ and $\quad H_{m} \equiv H\left(m_{h_{d}}\right)$. Additionally, in the second equality the temperature dependence on the effective number of relativistic degrees of freedom has been neglected. Note that determining the temperature from which the evolution described by Eq. (C4) holds, is nontrivial.

Within this approximation, Eq. (C4) provides an analytic solution for the DHDM number density,

$$
\frac{n}{s}=\mathcal{A}\left[1+3 r^{3 / 2} e^{-\Delta R_{\Gamma} x^{2}} \frac{\Gamma_{\gamma_{d}}^{0}}{{ }^{2 \Delta R_{\Gamma} H_{m}}-1},\right.
$$

by also using the equality $n_{h_{d}} / n=n_{h_{d}}^{\mathrm{eq}} / n^{\mathrm{eq}}$. The constant, $\mathcal{A}$, depends on the initial conditions at $x^{\Gamma}$. The above result is extremely informative. First, note that the total number of particles can only be reduced if $\Gamma_{\gamma_{d}}^{0} / 2 H_{m}>1$. Additionally, when $\Delta R_{\Gamma} x^{2} \gg 1$, the yield, $n / s$, goes to a constant, i.e., it becomes temperature independent. Thus, as long as the $h_{d} h_{d} \leftrightarrow \gamma_{d} \gamma_{d}$ cross section is large enough to ensure chemical equilibrium throughout the time where $\gamma_{d}$ decays are active, the final abundance does not depend on the magnitude of the cross section for this process. Note that this behavior differs significantly from the oneway/co-decay and forbidden scenarios considered in the literature. Essentially, the constant yield is the result of a balance between chemical equilibrium in the DS and the effect of $\gamma_{d}$ decay. The specific requirements for this mechanism to occur are a decaying state that is heavier than the DM, and a DS temperature that scales as $x_{d} \propto x^{2}$. Further analysis and possible generalizations will be considered in a future study.

The numerical solution of the BEs in this regime can differ significantly from the approximation given by Eq. (C5). This difference is mainly due to corrections to the scaling of the dark temperature, $T_{d}\left(T_{\mathrm{SM}}\right)$ (see Fig. 6 in the main text), and to the $T_{\mathrm{SM}}$ dependence on the effective number of degrees of freedom of the SM plasma; both of which were simplified in the derivation above. However the parametric dependence of this solution is reflected in the numerical results. Namely, the DHDM abundance is exponentially sensitive to the mass splitting $\Delta=r-1$, and to the kinetic mixing parameter $\epsilon$ (which controls the onset of $\gamma_{d}$ decay and therefore the value of $x^{\Gamma}$ ), while being almost independent of to the $h_{d} h_{d} \leftrightarrow \gamma_{d} \gamma_{d}$ cross section.
[1] M. Battaglieri, A. Belloni, A. Chou, P. Cushman, B. Echenard, R. Essig, J. Estrada, J. L. Feng, B. Flaugher, and P. J. Fox et al., arXiv:1707.04591.

[2] J. Beacham et al., J. Phys. G 47, 010501 (2020).

[3] B. Batell, M. Pospelov, and A. Ritz, Phys. Rev. D 79, 115008 (2009).

[4] L. Darmé, S. Rao, and L. Roszkowski, J. High Energy Phys. 03 (2018) 084.
[5] R. Essig, E. Kuflik, S. D. McDermott, T. Volansky, and K. M. Zurek, J. High Energy Phys. 11 (2013) 193.

[6] T. R. Slatyer and C.-L. Wu, Phys. Rev. D 95, 023010 (2017).

[7] V. Poulin, J. Lesgourgues, and P. D. Serpico, J. Cosmol. Astropart. Phys. 03 (2017) 043.

[8] A. Fradette, M. Pospelov, J. Pradler, and A. Ritz, Phys. Rev. D 90, 035022 (2014). 
[9] J. H. Chang, R. Essig, and S. D. McDermott, J. High Energy Phys. 01 (2017) 107.

[10] W. DeRocco, P. W. Graham, D. Kasen, G. Marques-Tavares, and S. Rajendran, J. High Energy Phys. 02 (2019) 171.

[11] M. Pospelov, A. Ritz, and M. B. Voloshin, Phys. Rev. D 78, 115012 (2008).

[12] J. Redondo and M. Postma, J. Cosmol. Astropart. Phys. 02 (2009) 005.

[13] L. J. Hall, K. Jedamzik, J. March-Russell, and S. M. West, J. High Energy Phys. 03 (2010) 080.

[14] N. Aghanim et al. (Planck Collaboration), Astron. Astrophys. 641, A6 (2020).

[15] J. Berger, K. Jedamzik, and D. G. E. Walker, J. Cosmol. Astropart. Phys. 11 (2016) 032.

[16] T. Hambye, M. H. G. Tytgat, J. Vandecasteele, and L. Vanderheyden, Phys. Rev. D 100, 095018 (2019).

[17] X. Chu, Y. Mambrini, J. Quevillon, and B. Zaldivar, J. Cosmol. Astropart. Phys. 01 (2014) 034.

[18] N. Bernal and X. Chu, J. Cosmol. Astropart. Phys. 01 (2016) 006.

[19] A. Falkowski, E. Kuflik, N. Levi, and T. Volansky, Phys. Rev. D 99, 015022 (2019).

[20] R. S. L. Hansen and S. Vogl, Phys. Rev. Lett. 119, 251305 (2017).

[21] H. An, R. Huo, and W. Liu, arXiv:1812.05699.

[22] R. N. Mohapatra and N. Okada, Phys. Rev. D 101, 115022 (2020).

[23] D. N. Spergel and P. J. Steinhardt, Phys. Rev. Lett. 84, 3760 (2000).
[24] E. D. Carlson, M. E. Machacek, and L. J. Hall, Astrophys. J. 398, 43 (1992).

[25] D. Pappadopulo, J. T. Ruderman, and G. Trevisan, Phys. Rev. D 94, 035005 (2016).

[26] M. Farina, D. Pappadopulo, J. T. Ruderman, and G. Trevisan, J. High Energy Phys. 12 (2016) 039.

[27] J. A. Dror, E. Kuflik, and W. H. Ng, Phys. Rev. Lett. 117, 211801 (2016).

[28] K. Griest and D. Seckel, Phys. Rev. D 43, 3191 (1991).

[29] R. T. D’Agnolo and J. T. Ruderman, Phys. Rev. Lett. 115, 061301 (2015).

[30] J. M. Cline, H. Liu, T. Slatyer, and W. Xue, Phys. Rev. D 96, 083521 (2017).

[31] J. A. Evans, S. Gori, and J. Shelton, J. High Energy Phys. 02 (2018) 100.

[32] J. Knodlseder et al., Astron. Astrophys. 411, L457 (2003).

[33] C. Picciotto and M. Pospelov, Phys. Lett. B 605, 15 (2005).

[34] N. Bar, K. Blum, and G. D’amico, Phys. Rev. D 101, 123025 (2020).

[35] P. A. R. Ade et al. (Planck Collaboration), Astron. Astrophys. 594, A13 (2016).

[36] D. Hooper and L.-T. Wang, Phys. Rev. D 70, 063506 (2004).

[37] M. Ibe, S. Kobayashi, Y. Nakayama, and S. Shirai, J. High Energy Phys. 04 (2020) 009.

[38] E. W. Kolb and M. S. Turner, Front. Phys. 69, 1 (1990).

[39] A. Belyaev, N. D. Christensen, and A. Pukhov, Comput. Phys. Commun. 184, 1729 (2013).

[40] P. Gondolo and G. Gelmini, Nucl. Phys. B360, 145 (1991). 